\title{
3-MANIFOLDS THAT ARE SUMS OF SOLID TORI AND SEIFERT FIBER SPACES
}

\author{
WOLFGANG HEIL ${ }^{1}$
}

\begin{abstract}
It is shown that a simply connected 3-manifold is $S^{3}$ if it is a sum of a Seifert fiber space and solid tori. Let $F$ be an orientable Seifert fiber space with a disk as orbit surface. It is shown that a sum of $F$ and a solid torus is a Seifert fiber space or a connected sum of lens spaces.

Let $M$ be a closed 3-manifold which is a union of three solid tori. It is shown that $M$ is a Seifert fiber space or the connected sum of two lens spaces (including $S^{1} \times S^{2}$ ).
\end{abstract}

Let $M$ be a sum of a Seifert fiber space and solid tori. It is shown (Theorem 1) that if $M$ is simply connected, then $M$ is $S^{3}$. This generalizes Hempel's result in [4]. Let $F$ be a Seifert fiber space with orbit surface a disk. A particular example is the complement (of a regular neighborhood in $S^{3}$ ) of a torus knot. The structure of all 3 manifolds $N$ that are a sum of $F$ and a solid torus is described (Theorem 3 ). Also the structure of all 3-manifolds that are a union of three solid tori (such that the intersection of any two is an annulus) is described (Theorem 4). In particular a question of A. C. Connor [3] is answered in the affirmative, that any such 3-manifold is $S^{3}$ if it is simply connected.

1. We work throughout in the piecewise linear category. A sum of a 3-manifold $F$ and solid tori $V_{1}, \cdots, V_{n}$ is the manifold obtained from $F$ and $V_{1}, \cdots, V_{n}$ by identifying components $T_{i}$ of $\partial F$ with $\partial V_{i}$ under homeomorphisms $f_{i}: \partial V_{i} \rightarrow T_{i}(i=1, \cdots, n)$. The connected sum $M_{1} \# M_{2}$ of two 3-manifolds is the manifold obtained by removing 3-balls in $\operatorname{int}\left(M_{1}\right)$ and $\operatorname{int}\left(M_{2}\right)$, and identifying the resulting 2-sphere boundaries under an orientation reversing homeomorphism. For a definition and classification of Seifert fiber spaces, see [5] and [6]. If $F$ is a Seifert fiber space there is a map $P$ of $F$ onto its orbit surface $f$ (Zerlegungsfläche). The image of an exceptional fiber is an exceptional point on $f$.

Received by the editors March 10, 1972 and, in revised form, May 9, 1972.

AMS (MOS) subject classifications (1970). Primary 57A10, 55A40.

Key words and phrases. Seifert fiber spaces, Dehn construction, simply connected 3manifold.

1 Partially supported by National Science Foundation grant GP-19964.

(c) American Mathematical Society 1973 
2. Simply connected 3-manifolds. Let $m_{i}$ be a meridian curve on $\partial V_{i}$ $\left(m_{i} \sim 0\right.$ in $V_{i}$ but $m_{i} \neq 0$ on $\left.\partial V_{i}\right)$.

Lemma 1. Suppose $M$ is a sum of a Seifert fiber space $F$ and solid tori $V_{i}$ $(i=1, \cdots, r ; r \geqq 2)$. If $f_{1}\left(m_{1}\right)$ and $f_{2}\left(m_{2}\right)$ are homologous (on $\partial V_{1}$ and $\partial V_{2}$ respectively) to a fiber, then $M$ contains a nonseparating 2-sphere.

Proof. We can assume that $f_{i}\left(m_{i}\right)$ is a fiber, $i=1,2$. Let $P$ be the projection of $F$ onto the orbit surface $f$ (Zerlegungsfläche). Choose a (nonseparating) arc $c$ on $f$ which does not meet exceptional points and which joins $P f_{1}\left(m_{1}\right)$ and $P f_{2}\left(m_{2}\right)$. Then $P^{-1}(c)$ is an annulus $A$ with boundary $f_{1}\left(m_{1}\right)$ and $f_{2}\left(m_{2}\right)$ which bound disks $D_{1}$ and $D_{2}$ in $\mathrm{Cl}(M-F)$. Thus $D_{1} \cup A \cup D_{2}$ is a nonseparating 2-sphere.

LEMMA 2. Suppose $M$ is a simply connected manifold which is a sum of a Seifert fiber space $F$ and a solid torus $V$ such that $f(m) \sim$ fiber (on $\partial F$ ) then $F$ is a solid torus (and without exceptional fibers).

Proof. Clearly $\partial F=\partial V$ and $F$ is orientable. We obtain a presentation for $\pi_{1}(F)$ as follows (see [7]). Let $D_{1}, \cdots, D_{r}$ be disjoint disks in the orbit surface $f$ such that $D_{i}$ contains the $i$ th exceptional fiber in its interior and such that $D_{i} \cap F$ is an arc in $\partial f(i=1, \cdots, r)$. Then $F^{\prime}=P^{-1}\left(\mathrm{Cl}\left(f-\cup D_{j}\right)\right)$ is a fiberbundle with fiber $S^{1}$. Therefore $\pi_{1}\left(F^{\prime}\right)=\left\{x_{1}, \cdots, x_{k}, h: x_{i}^{-1} h x_{i}=\right.$ $\left.h^{\varepsilon_{i}}, \varepsilon_{i}= \pm 1\right\}$ where $k=2 g$ if $f$ is orientable of genus $g$ and $k=g$ if $f$ is nonorientable of genus $g$. Now $V_{j}=P^{-1}\left(D_{j}\right)$ is a solid torus, the image of $\pi_{1}\left(F^{\prime} \cap V_{j}\right)$ in $\pi_{1}\left(F^{\prime}\right)$ is $\{h\}$ and the image in $\pi_{1}\left(V_{j}\right)$ has index $\mu_{i}$, where $\left|\mu_{i}\right| \geqq 2$ (the order of the $i$ th exceptional fiber). Therefore

$$
\pi_{1}(F)=\left\{x_{1}, \cdots, x_{k}, y_{1}, \cdots, y_{r}, h: x_{i}^{-1} h x_{i}=h^{\varepsilon_{i}}, y_{j}^{\mu_{j}}=h\right\} .
$$

$\pi_{1}(M)$ is obtained from $\pi_{1}(F)$ by adding the relation $h=1$, and is a free product of $k+r$ nontrivial cyclic factors. Therefore $\pi_{1}(M)=1$ implies that $k=r=0$, which means that the orbit surface $f$ is a disk without exceptional points and $F=P^{-1}(f)$ is a fibered solid torus without exceptional fibers.

THEOREM 1. A simply connected 3-manifold is $S^{3}$ if it is a sum of a Seifert fiber space $F$ and solid tori.

Proof. Since $\partial F$ has no 2-sphere components and since $\pi_{1}(M)=1$, it follows that $M$ is closed. Therefore $\partial F$ consists of tori $T_{i}$ which are to be identified with the boundary of solid tori $V_{i}(i=1, \cdots, n)$. Let $m_{i}$ be a meridian curve on $\partial V_{i}$. If, for each $i=1, \cdots, n, f_{i}\left(m_{i}\right)$ is not homologous to a fiber on $T_{i}$, then the fibering on $T_{i}$ can be extended to a fibering on $V_{i}$. Then $M$ is a Seifert fiber space. By [5, Satz 11], $M$ is $S^{3}$. If $f_{i}\left(m_{i}\right)$ is homologous to a fiber for at least two $i$, then $M$ cannot be simply connected by 
Lemma 1. Therefore assume $f_{1}\left(m_{1}\right) \sim$ fiber, but $f_{i}\left(m_{i}\right) \nsucc$ fiber for $i=2, \cdots$, $n$. Let $M^{\prime}$ be the sum of $F$ and the solid tori $V_{i}(i=2, \cdots, n)$. Then $M^{\prime}$ is a Seifert fiber space and therefore a solid torus by Lemma 2. (In fact the proof of Lemma 2 shows that $F$ is already a solid torus.) It follows that $M=M^{\prime} \cup V_{1}$ is $S^{3}$.

COROLlary [4]. A simply connected 3-manifold is $S^{3}$ if it is a sum of a solid torus and the complement of a torus knot.

Corollary 1. A simply connected 3-manifold is $S^{3}$ if it is a sum of solid tori and the complement of a link in $S^{3}$ whose group has nontrivial center.

Proof. It follows from [7] that the complement of such a link is a Seifert fiber space. For a classification of these links see [1].

Theorem 2. A simply connected 3-manifold is $S^{3}$ if it is a union of three solid tori (such that the intersection of any two of them is an annulus).

Proof. Let $M=V_{1} \cup V_{2} \cup V_{3}$, a sum of three solid tori and let $V_{i} \cap V_{j}=$ $A_{i j}(i, j=1,2,3, i \neq j)$ an annulus. Let $a$ be a component of $A_{12} \cap A_{13} \cap A_{23}$ and let $m_{i}$ on $\partial V_{i}$ be a meridian of $V_{i}(i=1,2,3)$.

If $a \nsucc m_{1}$ and $a \nsucc m_{2}$ on $\partial V_{1}$ and $\partial V_{2}$, respectively, we can fiber $V_{1}$ and $V_{2}$ (in the sense of Seifert) such that $a$ is a (ordinary) fiber. Then $N=V_{1} \cup$ $V_{2}$ is a Seifert fiber space and $M$ is a sum of $N$ and the solid torus $V_{3}$. By Theorem 1 , if $M$ is simply connected, then $M$ is $S^{3}$. The same argument applies if $a \nsucc m_{1}, a \nsucc m_{3}$ and if $a \nsucc m_{2}, a \nsucc m_{3}$.

Therefore we can assume that $a \sim m_{1}$ on $\partial V_{1}$ and $a \sim m_{2}$ on $\partial V_{2}$. But then $\pi_{1}(N)=Z * Z$ and $\pi_{1}(M)$ is obtained from $\pi_{1}(N)$ by adding one relation (which describes the curve on $\partial N$ that is identified with a meridian $m_{3}$ on $\left.\partial V_{3}\right)$. Therefore $\pi_{1}(M)$ cannot be trivial, a contradiction.

3. Sums of some Seifert fiber spaces and solid tori. A lens space is a sum of two solid tori different from $S^{1} \times S^{2}$. We say a lens space is nontrivial if it is not $S^{3}$.

LEMMA 3. Let $F$ be an orientable Seifert fiber space with $r \geqq 1$ exceptional fibers. Suppose the orbit surface is a disk. Let $A$ be an annulus on $\partial F$ consisting of fibers. If $M$ is the manifold obtained from $F$ by adding a 2-handle along $A$, then $M$ is the connected sum of $r$ nontrivial lens spaces minus an open 3-ball.

Proof. If $r=1$ then $F$ is a solid torus and the theorem follows from the classification of Heegaard splittings of genus 1 . Suppose the theorem is true for $r=n-1$ and suppose $F$ has $n$ exceptional fibers. Decompose the orbit surface $f$ into two disks $d, d^{\prime}$ such that int $(d)$ and int $\left(d^{\prime}\right)$ contain one and $n-1$ exceptional points respectively, and $d \cap d^{\prime}$ is an arc $c$ with $c \cap \partial f=\partial c$ 
consisting of two points $h_{1}, h_{2}$ such that $h_{1} \in \operatorname{int}(P(A)), h_{2} \notin P(A), h_{1}, h_{2}$ separate $\partial f$ into two arcs $l, l^{\prime}$ such that $\partial d=l \cup c, \partial d^{\prime}=l^{\prime} \cup c$. Let $a=P(A) \cap$ $d, a^{\prime}=P(A) \cap d^{\prime}$. Let $V=P^{-1} d, F^{\prime}=P^{-1} d^{\prime}$. The 2-handle $B$ in $M$ is decomposed into two 3-balls $B_{1}, B_{2}$ where the annuli $P^{-1} a$ on $B_{1}$ and $P^{-1} a^{\prime}$ on $B_{2}$ constitute the annulus $A$ on $B$ and $B_{1} \cap B_{2}=D$, a disk with $\partial D=$ $P^{-1}\left(h_{1}\right)$. Let $M_{1}=V \cup B_{1}, M_{2}=F^{\prime} \cup B_{2}$. Clearly the hypothesis of the theorem applies to $M_{1}, M_{2}$; therefore $M_{1}$ is a lens space minus an open 3-ball and $M_{2}$ is the connected sum of $n-1$ lens spaces minus an open 3-ball. But $M=M_{1} \cup M_{2}, M_{1} \cap M_{2}=\partial M_{1} \cap \partial M_{2}=\left(P^{-1} c\right) \cup D$, a disk. It follows that $M$ is the disk sum of $M_{1}$ and $M_{2}$ and hence that $M$ is $M_{1} \# M_{2}$ minus an open 3-ball.

THEOREM 3. Let $F$ be an orientable Seifert fiber space with $r \geqq 1$ exceptional fibers and a disk as orbit surface. If $M$ is a sum of $F$ and a solid torus $V$, then $M$ is a Seifert fiber space (with $r$ or $r+1$ exceptional fibers and $a$ 2-sphere as orbit surface) or the connected sum of $r$ (nontrivial) lens spaces.

Proof. If the fibering of $F$ cannot be extended to a fibering of $M$ then a fiber $h$ in $\partial M$ is a meridian of $V$ and therefore the theorem follows from Lemma 3.

COROLlaRY 2. If $F$ is the complement (of a regular neighborhood in $S^{3}$ ) of a torus knot and if $M$ is a sum of $F$ and a solid torus $V$, then $M$ is a Seifert fiber space (with at most three exceptional fibers and a 2-sphere as orbit surface) or the connected sum of two (nontrivial) lens spaces.

Thus in particular any such manifold is in the "Poincare category" (i.e. every embedded homotopy 3-cell is a 3-cell).

4. Sums of solid tori. We now generalize Theorem 2 as follows:

THEOREM 4. Let $M$ be a closed 3-manifold which is a union of three solid tori (such that the intersection of any two of them is an annulus). Then $M$ is either a Seifert fiber space (with orbit surface a 2-sphere and at most three exceptional fibers), or the connected sum of two (nontrivial) lens spaces, or the connected sum of a lens space and $S^{1} \times S^{2}$ or $\left(S^{1} \times S^{2}\right) \#\left(S^{1} \times S^{2}\right)$.

Proof. Refer to the proof of Theorem 2, $M=V_{1} \cup V_{2} \cup V_{3}$. If $N=$ $V_{1} \cup V_{2}$ is a Seifert fiber space the result follows from Theorem 3. Similarly if $V_{2} \cup V_{3}$ or $V_{1} \cup V_{3}$ are Seifert fiber spaces.

If $a \sim m_{1}$ on $\partial V_{1}$ and $a \sim m_{2}$ on $\partial V_{2}$ then it is not hard to see that $N=$ $V_{1} \cup V_{2}$ is the connected sum of a solid torus and $S^{1} \times S^{2}$. Therefore $M$ is the connected sum of a lens space and $S^{1} \times S^{2}$ or $\left(S^{1} \times S^{2}\right) \#\left(S^{1} \times S^{2}\right)$. 
5. Unions of two solid tori. Consider the union of two solid tori $V_{1}, V_{2}$ along an annulus $A$ (which is not meridional on $V_{1}$ and $V_{2}$ ). Fibering $V_{1}$ and $V_{2}$ such that $\partial A$ is a fiber, we obtain a Seifert fiber space $N$ with invariants $\alpha_{1}, \beta_{1} ; \alpha_{2}, \beta_{2}\left(\alpha_{i} \geqq 2,0<\beta_{i}<\alpha_{i}\right)$ and fundamental group $G=$ $\left\{t_{1}, t_{2}: t_{1}^{\alpha_{1}}=t_{2}^{\alpha_{2}}\right\}$. If $N^{\prime}$ is another such space with invariants $\alpha_{1}, \beta_{1}^{\prime} ; \alpha_{2}, \beta_{2}^{\prime}$ then it follows from [6] that $N$ is not homeomorphic to $N^{\prime}$ if $\beta_{i}^{\prime} \neq \beta_{i}$. Therefore (for $\alpha_{1}>3$ ) this construction gives us 3-manifolds with boundary a torus which are not homeomorphic but which have the same fundamental group $G$, the group of a torus knot. Such manifolds have also been obtained by $\mathrm{H}$. Zieschang [8] by considering certain Heegaard splittings of genus 2.

I thank the referee for suggesting the following theorem.

THEOREM 5. Let $M$ be a union of two solid tori along an annulus. $M$ is the complement (of a regular neighborhood in $S^{3}$ ) of a torus knot (including the unknot) if and only if $M$ can be embedded in a simply connected 3-manifold $N$.

Proof. We may assume that $M$ is not a solid torus. Therefore $\pi_{1}(\partial M) \rightarrow \pi_{1}(M)$ is an injection. Let $V=\mathrm{Cl}(N-M)$. If $\pi_{1}(\partial V) \rightarrow \pi_{1}(V)$ is an injection then $N=V \cup M$ would not be simply connected. But if $\pi_{1}(\partial V) \rightarrow$ $\pi_{1}(V)$ is not an injection then $V$ is a fake solid torus, i.e. $V$ is obtained by identifying a pair of disjoint disks in the boundary of a homotopy 3-ball. For by Dehn's lemma and the Loop theorem there is a disk $D$ in $V$ such that $\partial D=\partial V \cap D$ is not contractible on $\partial V$. Cutting $V$ along $D$ we obtain a submanifo'd of $N$ with boundary a 2-sphere, which is a homotopy 3ball. Let $m=\partial D$. This is a simple closed curve on $\partial M$ and $\pi_{1}(M)$ becomes trivial by adding a 2-handle along an annular neighborhood of $m$ on $\partial M$. Filling in the resulting 2-sphere with a 3-ball we obtain a simply connected 3-manifold which is a sum of $M$ and a solid torus. By Theorem 2, $M$ is the complement of a regular neighborhood of a knot in $S^{3}$. Since $\pi_{1}(M)$ has a nontrivial center, it is a torus knot [2].

ADDED IN PROOF. A detailed version of Corollary 2 of this paper has been obtained by L. Moser [9].

\section{REFERENCES}

1. G. Burde and K. Murasugi, Links and Seifert fiber spaces, Duke Math. J. 37 (1970), 89-93. MR 40 \#6528.

2. G. Burde and $\mathrm{H}$. Zieschang, Eine Kennzeichnung der Torusknoten, Math. Ann. 167 (1966), 169-176. MR 35 \#1008.

3. A. C. Connor, Partially splittable knots (to appear).

4. J. Hempel, A simply connected 3-manifold is $S^{3}$ if it is the sum of a solid torus and the complement of a torus knot, Proc. Amer. Math. Soc. 15 (1964), 154-158. MR 28 \#599. 
5. H. Seifert, Topologie dreidimensionaler gefaserter Räume, Acta Math. 60 (1933), 147-238.

6. P. Orlik, E. Vogt and H. Zieschang, Zur Topologie gefaserter dreidimensionaler Mannigfaltigkeiten, Topology 6 (1967), 49-64. MR 35 \#3696.

7. F. Waldhausen, Gruppen mit Zentrum und 3-dimensionale Mannigfaltigkeiten, Topology 6 (1967), 505-517. MR 38 \#5223.

8. H. Zieschang, Über einfache Kurvensysteme auf einer Vollbrezel vom Geschlecht 2, Abh. Math. Sem. Univ. Hamburg. 26 (1964), 237-247. MR 28 \#4524.

9. L. Moser, Elementary surgery along a torus knot, Pacific J. Math. 38 (1971), 737-745.

Department of Mathematics, Florida State University, Tallahassee, Florida 32306 